University of Nebraska - Lincoln

DigitalCommons@University of Nebraska - Lincoln

January 1996

\title{
Scanning force microscopy for the study of domain structure in ferroelectric thin films
}

Alexei Gruverman

University of Nebraska-Lincoln, agruverman2@unl.edu

O. Auciello

MCNC, Electronics Technologies Division, Research Triangle Park, North Carolina

H. Tokumoto

Joint Research Center for Atom Technology, National Institute for Advanced Interdisciplinary Research, Tsukuba, Ibaraki 305, Japan

Follow this and additional works at: https://digitalcommons.unl.edu/physicsgruverman

Part of the Physics Commons

Gruverman, Alexei; Auciello, O.; and Tokumoto, H., "Scanning force microscopy for the study of domain structure in ferroelectric thin films" (1996). Alexei Gruverman Publications. 36.

https://digitalcommons.unl.edu/physicsgruverman/36

This Article is brought to you for free and open access by the Research Papers in Physics and Astronomy at DigitalCommons@University of Nebraska - Lincoln. It has been accepted for inclusion in Alexei Gruverman Publications by an authorized administrator of DigitalCommons@University of Nebraska - Lincoln. 


\title{
Scanning force microscopy for the study of domain structure in ferroelectric thin films
}

\author{
A. Gruverman a) \\ Joint Research Center for Atom Technology, National Institute for Advanced Interdisciplinary Research, \\ Tsukuba, Ibaraki 305, Japan \\ O. Auciello \\ MCNC, Electronics Technologies Division, Research Triangle Park, North Carolina 27709-2889 \\ H. Tokumoto \\ Joint Research Center for Atom Technology, National Institute for Advanced Interdisciplinary Research, \\ Tsukuba, Ibaraki 305, Japan
}

(Received 10 October 1995; accepted 22 December 1995)

\begin{abstract}
A piezoresponse technique based on scanning force microscopy (SFM) has been used for studying domain structure in ferroelectric thin films. Studies were performed on $\mathrm{Pb}\left(\mathrm{Zr}_{x}, \mathrm{Ti}_{1-x}\right) \mathrm{O}_{3}(\mathrm{PZT})$ thin films produced by a sol-gel method. The piezoresponse images of the PZT films were taken before and after inducing polarization in the films by applying a direct current voltage between the bottom electrode and the SFM tip. Polarization induced patterns were written with $20 \mathrm{~V}$ pulses and subsequently imaged by the SFM piezoresponse technique. The effect of the film structure on the imaging resolution of domains is discussed. (C) 1996 American Vacuum Society.
\end{abstract}

\section{INTRODUCTION}

Ferroelectric materials in thin layer form are being intensively investigated for application to many advanced devices such as nonvolatile memories, sensors, and actuators. However, some problems, such as electrical degradation, fatigue and aging, so far have limited the wide application of ferroelectrics in microelectronic devices. Although the fatigue effect can be practically eliminated in ferroelectric capacitors using appropriate electrodes, ${ }^{1-3}$ work is still necessary to better understand this phenomenon. The currently available data indicate that control of domain structure is critical to achieving the optimal physical properties of ferroelectric thin films. ${ }^{4,5}$ In this article we describe the use of scanning force microscopy (SFM) for the investigation of the domain structure in $\mathrm{Pb}\left(\mathrm{Zr}_{x} \mathrm{Ti}_{1-x}\right) \mathrm{O}_{3}(\mathrm{PZT})$ thin films.

SFM was successfully applied for the visualization and control of domain structure in ferroelectric crystals of gadolinium molybdate, ${ }^{6}$ guanidinium aluminum sulphate hexahydrate, ${ }^{7-10}$ and triglycine sulphate (TGS). ${ }^{8,11}$ An advantage of the SFM technique over other well-known methods, such as scanning electron microscopy, optical polarizing microscopy, and surface decoration technique, ${ }^{12}$ is that SFM combines high spatial resolution ${ }^{13}$ with the ability to yield data from the analysis of samples in ambient environment and without the need for special sample preparation. Recently, Franke et al. reported the use of scanning force microscopy for the detection and modification of domains in ferroelectric PZT thin films prepared by sputter deposition. ${ }^{14}$ In the work reported in this article, a similar technique, based on the piezoelectric modulation of a ferroelectric layer, was used to study the domain structure in PZT films produced by a sol-gel technique. This work provided the opportunity of

a) On leave from the Institute of Physics and Applied Mathematics, Ural State University, Ekaterinburg 620083, Russia. comparing the switching behavior in ferroelectric films produced by different techniques and to clarify the effect of the film structure on the imaging resolution, which can be achieved during SFM visualization of ferroelectric domains. In addition, the work described here demonstrates the applicability of the SFM method for writing and imaging complex domain patterns in a micrometer scale.

\section{EXPERIMENTAL APPROACH}

A commercial force microscope (Seiko Instruments SPA 300) was used in this study. To apply an external field we used a standard gold coated $\mathrm{Si}_{3} \mathrm{~N}_{4}$ cantilever (Olympus Opt. Inc.) with a spring constant $0.09 \mathrm{~N} / \mathrm{m}$, a resonant frequency of $36 \mathrm{kHz}$, and an integrated tip of about $50 \mathrm{~nm}$ in diameter. Experiments were carried out using two samples involving $\mathrm{Pb}\left(\mathrm{Zr}_{0.53}, \mathrm{Ti}_{0.47}\right) \mathrm{O}_{3}$ ferroelectric layers. Two capacitor structures were produced:

(1) A Pt/0.8 $\mu \mathrm{m} \mathrm{PZT/Pt} \mathrm{heterostructure,} \mathrm{hereafter} \mathrm{desig-}$ nated as PZT-0.8, fabricated on a (100) MgO substrate, where the Pt bottom and top electrodes were synthesized using an ion-beam sputter-deposition method, ${ }^{1}$ and the PZT layer was produced using a sol-gel processing technique.

(2) $\mathrm{A} \mathrm{RuO}_{2} / 0.18 \mu \mathrm{m} \mathrm{PZT} / \mathrm{RuO}_{2}$ heterostructure fabricated on $\mathrm{a} \mathrm{SiO}_{2} / \mathrm{Si}$ substrate, hereafter designated PZT-0.18, where the electrodes and PZT layers were produced using the same techniques as for the PZT- 0.8 capacitor.

It is well known that the application of a voltage pulse to the PZT film results in a change of its thickness. The SFM imaging method is based on the detection of the film vibration caused by the external ac field applied to the film through the SFM tip. Due to the strong coupling forces between the tip and sample surface, which are kept constant by the feedback loop, the cantilever vibrates together with the 
film, following the surface oscillations. ${ }^{13,14}$ The deflection signal from the cantilever is detected by a lock-in amplifier, which output signal provides information about the change in the film thickness at any instant of the film vibration. Moreover, the phase of the output signal depends on the sign of the piezoelectric coefficient (and therefore on the polarization direction) and reverses when the coefficient is opposite. As a result, the regions with opposite orientation of polarization can be imaged without being influenced by topographical roughness. In the present experiment, the PZT film was imaged by applying a small ac voltage with an amplitude of $1-3 \mathrm{~V}$ and a frequency of $5 \mathrm{kHz}$ between the SFM tip and the bottom Pt electrode. A number of dc voltages of various amplitudes and polarities were applied to the films through the SFM tip to induce the local polarization of the film. The voltage applied to the film depends essentially on the tip-sample distance, so the tip was kept in gentle mechanical contact with the surface to provide the maximum strain signal during the measurements. In this way the topography image of the film surface was taken together with the domain imaging.

The ac electric field with a frequency $f$ causes a film vibration with the same frequency due to the piezoelectric effect and with a frequency $2 f$ due to electrostriction. The film response due to the piezoelectric effect prevails when the polarization vector is normal to the film surface and does not change its sign from grain to grain in the direction of the film thickness. The applied electric field compresses grains with a given direction of polarization and expands grains with opposite polarization. The amplitude and the phase of the detected signal, integrated over the entire range of the film thickness, provide the information about the integral strain induced along the film thickness and about the direction of the polarization, respectively. If all grains in the direction over the film thickness are polarized randomly, the piezoelectric response cannot be observed, because the expansion of one grain, with polarization component along the external field, will be compensated by the compression of another grain, with polarization component opposite to the applied field. Therefore, only the strain which is proportional to the square of the electric field, i.e., electrostrictive response, could be detected. If the applied ac field is high enough to change the polarization direction in some grains, the signals from the domain reorientation will contribute to the detected signal causing a phase shift and an increase in the piezoelectric component of the integral response.

\section{RESULTS AND DISCUSSION}

Analysis of the electric response of the film showed that the as-processed film was in the nonpolar state, since the response was of frequency $2 f$, which can be associated with electrostriction. No change in the piezoresponse image was observed after $30 \mathrm{~min}$ of scanning over the same area, which indicates that the applied ac voltage of $3 \mathrm{~V}$ does not affect the polarization during the image acquisition.

Figures 1(a) and 1(b) show topographic and piezoresponse images of the PZT-0.8 film, respectively, taken simul-
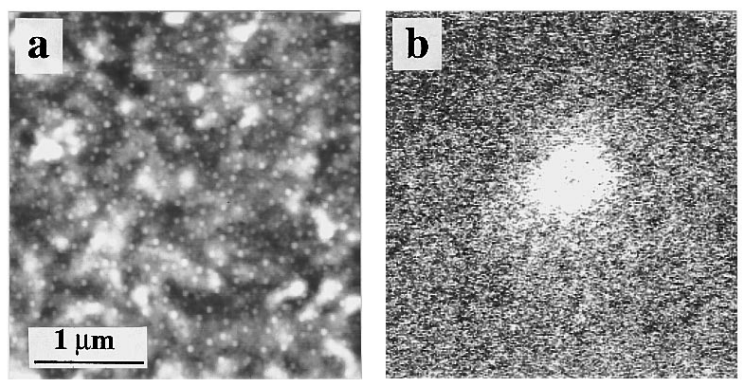

FIG. 1. Simultaneously obtained topographic (a) and piezoresponse (b) images of the PZT film with a scanning area of $3.5 \times 3.5 \mu \mathrm{m}^{2}$. The polarized region, produced by applying a positive voltage of $20 \mathrm{~V}$ for $1 \mathrm{~min}$, appears as a bright spot in the piezoresponse image (b).

taneously after the central part was polarized with a positive voltage pulse of $20 \mathrm{~V}$ applied for $1 \mathrm{~min}$ by the fixed SFM tip. The topography image reveals quite a rough surface with a topography variation of about $20 \mathrm{~nm}$. The applied field showed almost no effect on the topography image, whereas a result of the field application can be clearly seen in the piezoresponse image. The polarized region in Fig. 1(b) appeared as a bright spot because of its high vibration amplitude (about $0.5 \mathrm{~nm}$ ) in phase with the applied electric field. The diameter of the polarized region is about $700 \mathrm{~nm}$, suggesting that the electric field generated in the film by the tip is effectively extended to an area greater than that corresponding to the tip diameter (which is less than $100 \mathrm{~nm}$ ). Mechanical stresses, induced in the lateral direction during the reorientation of domains underneath the tip, can also cause the partial polarization of the film outside the tip.

The field-time dependence of the size of the polarized region, produced by the applied voltage, was measured to determine the threshold field and the lateral resolution limit for the polarization process. It was found that any noticeable polarization can be produced only by voltage pulses of more than $10 \mathrm{~V}$, while lower voltages are not sufficiently high as to polarize the film. Long exposure to a suitable electric field resulted in the increase of the size of the polarized region to a saturation value of about $900 \mathrm{~nm}$, which is of the order of the film thickness.

It is relevant to point out that the polarizing experiments can be more easily performed using a previously poled film. The threshold voltage in this case is about $7 \mathrm{~V}$. The smallest stable domain of reversed polarity written by the fixed tip in this case was about $200 \mathrm{~nm}$ in diameter [a dark spot in the center of the bright area in Fig. 2(a)], which is much smaller than that in the nonpolarized film. This can be understood if we take into account that in the previously polarized film the polarization change mainly proceeds through 180 domain switching which does not involve a strain change and hence does not affect the neighbors. Therefore, an effectively smaller area is involved in the switching process.

Particular attention was paid to the resolution power of the SFM piezoresponse technique. A cross-section analysis of the polarized region in the PZT- 0.8 sample revealed that the width of a transition region between the positive and 

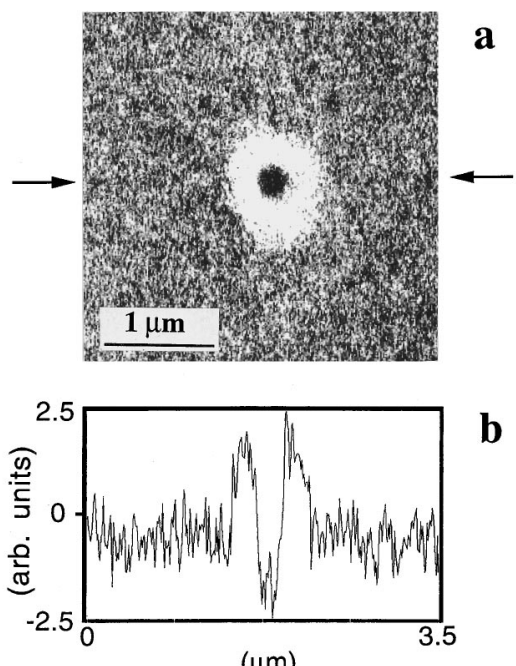

b

$(\mu \mathrm{m})$

FIG. 2. (a) Piezoresponse image of a PZT- 0.8 film excited by voltage pulses of opposite polarities. A positive $25 \mathrm{~V}$ voltage, applied to the central part of the film for $20 \mathrm{~s}$, resulted in a bright spot of $900 \mathrm{~nm}$ in diameter, corresponding to a polarized area. An opposite domain, which appeared as a dark spot of $300 \mathrm{~nm}$ in diameter, was subsequently written in by a negative $8 \mathrm{~V}$ pulse applied for $3 \mathrm{~s}$. (b) Cross section along the line marked by arrows on the piezoresponse image.

negative domains was about $80 \mathrm{~nm}$ [see Fig. 2(b)]. On the other hand, we have imaged $c$ domains in barium titanate single crystal, using the same SFM piezoresponse technique, with the resolution of $30 \mathrm{~nm}$, which was of the order of the tip radius. ${ }^{15}$ Franke et al. reported achieving a better than 10 $\mathrm{nm}$ resolution when imaging a PZT film with columnar structure using a tip smaller than $20 \mathrm{~nm}$ diameter. ${ }^{14}$ Therefore, we conclude that the imaging resolution of $80 \mathrm{~nm}$ achieved for the PZT-0.8 film in the work reported here is due to the intrinsic properties of the film and not due to the resolution limit of the SFM piezoresponse technique itself, which can be as small as the tip radius. To confirm this hypothesis, we applied the SFM piezoresponse method to the PZT-0.18 film, where the domain structure similar to that in Fig. 2(a) was written and subsequently imaged. In this case, the transition width of the piezoresponse signal across the domain boundary was found to be about $30 \mathrm{~nm}$.

In the film with columnar structure used in Ref. 14, with crystallites extended from one surface to the opposite one, a situation of a single polarized crystallite could be easily realized. In this case, the imaging resolution of a domain structure will correspond to that of topographical features. In the case of the comparatively thick PZT- 0.8 film studied here there might be several grains in the direction of the film thickness, such that each grain in this direction, normal to the film surface, will be polarized differently with an applied electric field. Therefore, it is difficult to produce a sharp boundary between two polarized regions and as a result a transition region on the piezoresponse image of the PZT- 0.8 film will be more diffuse. On the other hand, we can expect a polarization reversal inside a single grain for the comparatively thin PZT-0.18 film, in which the single grain size is practically the same as the film thickness. This case is

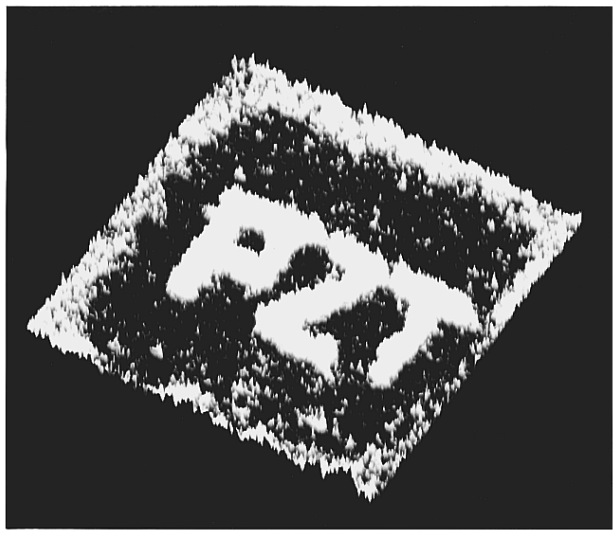

FIG. 3. Three-dimensional image of a polarization-induced "PZT" pattern written in the PZT- 0.8 film. A dark square of $4 \times 4 \mu \mathrm{m}^{2}$ was produced by scanning the SFM tip while applying a positive $20 \mathrm{~V}$ pulse. The "PZT" film character seen in the figure, produced by moving the SFM tip while applying a negative $10 \mathrm{~V}$ signal, exhibit opposite piezoelectric coefficients and opposite polarities (positive and negative, respectively). The gray region corresponds to a nonpolarized area, which does not show piezoelectric response. The scanning area is $5 \times 5 \mu \mathrm{m}^{2}$.

equivalent to the case of a single crystal where the resolution is mainly determined by the tip size, rather than by the intrinsic width of the domain wall which can be of the size of several lattice parameters.

Complex domain patterns can be written and imaged using the SFM piezoresponse technique. Figure 3 shows the piezoresponse image of the PZT- 0.8 film where a square area of $4 \times 4 \mu \mathrm{m}^{2}$ was polarized by scanning the tip while applying a positive $20 \mathrm{~V}$ excitation. Subsequently, a "PZT" domain pattern was written by the tip held under a negative 10 $\mathrm{V}$ dc pulse. The black area shown in Fig. 3 exhibits a positive piezoelectric constant and vibrates with relatively high amplitude in phase with the applied ac field. On the other hand, the bright "PZT" region has a negative piezoelectric constant and oscillates in counterphase with the ac field. The gray regions correspond to nonpolarized areas and do not exhibit any piezoelectric response. A relevant aspect of the data shown in Fig. 3 is that this polarization-induced pattern is stable, since no change was observed when imaging the same area about $10 \mathrm{~h}$ after the pattern was originally written.

Preliminary measurements have been performed to obtain the $d_{33}$ piezoelectric constant of the polarized PZT film using the SFM technique. Values in the range of $70-100 \times 10^{-12}$ $\mathrm{m} / \mathrm{V}$ have been measured, which are in a good agreement with values obtained by other methods. ${ }^{16}$ Further work is currently underway to refine the measurements.

In conclusion, a writing/imaging technique based on scanning force microscopy has been used for the study and control of domain structure in ferroelectric thin films. Experiments involved studies of PZT thin films of different thicknesses produced by a sol-gel method. Complex domain patterns were written by applying a dc voltage and were subsequently imaged by detecting the piezoelectric vibration of the surface induced by the ac electric field applied to the 
film through the SFM tip. The achieved imaging resolution depends on the film thickness and it is limited down to the tip radius. The obtained results showed that the nanoscale study of ferroelectric and piezoelectric properties of thin films can be effectively provided by the SFM technique. Work is in progress to obtain further data needed to understand the domain formation dynamics in PZT thin films.

\section{ACKNOWLEDGMENTS}

The authors acknowledge fruitful discussions with Dr. K. Takata and Dr. O. Kolosov. This work was supported by NEDO, Japan and by ARPA, USA. One of the authors (O. A.) thanks Dr. A. I. Kingon for facilitating the use of sol-gel film synthesis facilities.

${ }^{1}$ O. Auciello, K. D. Gifford, D. J. Lichtenwalner, R. Dat, H. N. Al-Shareef, K. R. Bellur, and A. I. Kingon, Integrated Ferroelect. 6, 173 (1995).

${ }^{2}$ R. Dat, D. J. Lichtenwalner, O. Auciello, and A. I. Kingon, Appl. Phys. Lett. 64, 2673 (1994).

${ }^{3}$ R. Ramesh, O. Auciello, V. G. Keramidas, and R. Dat, Science and Technology of Electroceramic Thin Films, edited by O. Auciello and R. Waser, NATO/ARW Series (Kluwer, Academic, Dordrecht, 1994), Vol. 284, p. 1.
${ }^{4}$ J. S. Speck, A. Seifert, W. Pompe, and R. Ramesh, J. Appl. Phys. 76, 477 (1994).

${ }^{5}$ W. L. Warren, D. Dimos, B. A. Tuttle, R. D. Nasby, and G. E. Pike, Appl. Phys. Lett. 65, 1018 (1994).

${ }^{6}$ F. Saurenbach and B. D. Terris, Appl. Phys. Lett. 56, 1703 (1990).

${ }^{7}$ R. Luthi, H. Haefke, K.-P. Meyer, E. Meyer, L. Howald, and H.-J. Guntherodt, J. Appl. Phys. 74, 7461 (1993).

${ }^{8}$ R. Luthi, H. Haefke, W. Gutmannsbauer, E. Meyer, L. Howald, and H.-J. Guntherodt, J. Vac. Sci. Technol. B 12, 2451 (1994).

${ }^{9}$ O. V. Kolosov, A. L. Gruverman, J. Hatano, K. Takahashi, and H. Tokumoto, Phys. Rev. Lett. 74, 4309 (1995).

${ }^{10}$ A. L. Gruverman, O. V. Kolosov, J. Hatano, K. Takahashi, and H. Tokumoto, J. Vac. Sci. Technol. B 13, 1095 (1995).

${ }^{11}$ M.-K. Bae, T. Horiuchi, K. Hara, Y. Ishibashi, and K. Matsushige, Jpn. J. Appl. Phys. 33, 1390 (1994).

${ }^{12}$ M. E. Lines and A. M. Glass, Principles and Applications of Ferroelectrics and Related Materials (Clarendon, Oxford, 1977).

${ }^{13}$ G. Binnig, C. F. Quate, and Ch. Gerber, Phys. Rev. Lett. 56, 930 (1986).

${ }^{14}$ K. Franke, J. Besold, W. Haessler, and C. Seegebarth, Surf. Sci. Lett. 302, L283 (1994).

${ }^{15}$ A. L. Gruverman, O. Auciello, J. Hatano, and H. Tokumoto, presented at the 8th European Meeting on Ferroelectricity, EMF8, Nijmegen, The Netherlands, 1995.

${ }^{16}$ J.-F. Li, D. D. Viehland, T. Tani, C. D. E. Lakeman, and D. A. Payne, J. Appl. Phys. 75, 442 (1994). 